\title{
Broadcast digital asset management at Lifetime Television
}

\section{Carl Charleson}

has 18 years of experience developing business applications. Currently he is Director of Digital Media Applications at Lifetime Entertainment. He is managing the development of a new digital asset management system for long form and promo content. Prior to joining Lifetime, he was in charge of affiliate systems at A\&E Television Networks.

Keywords: media asset management (MAM), digital asset management (DAM), IT, broadcast, development, project management

Abstract The merging of IT and broadcast technology has been an ever-expanding phenomenon. This merge is particularly evident in the area of digital asset management (DAM). The author, an experienced software developer, describes his first foray into the arena of broadcast technology. Currently he is working on a new DAM system for long form and promo content. The current post-production process will be replaced with an automated tapeless environment supporting ingest, quality control, dubbing, transfer to the editing system, archive management and transfer to on-air systems. This paper describes the education of the author on broadcast technology and the unique needs and concerns of his post-production clients. The merging of IT and broadcast, and the contributions each brings to the success of the implementation of a DAM system is set against the backdrop of a project as it progresses from high-level analysis, vendor selection, system design, hardware implementation, software development, testing and detailed workflow analysis. The author presents a project in the midst of development, with the recurrent pattern of successes and setbacks.

\section{THE FIRST DAY AT SCHOOL}

I was sitting in a meeting, my eyes following the conversation like an avid tennis fan watching Venus Williams and Lindsay Davenport at match point. I tried to interrupt the volley and ask a question, but the conversation quickly bounced in another direction. Having lost the moment, and not wanting to bring the meeting to a screeching halt, I quickly jotted down the acronym for a future Google search.

It was August 2004 and it was my first day at Lifetime Entertainment. I was sitting in a meeting with the head of the broadcast facility and several folks from post-production. We were discussing moving our broadcast facility from Astoria, Queens to a yet-to-be-built facility in downtown Manhattan. Along with that, we planned to upgrade all broadcast operations and postproduction systems and IT infrastructure. It was also an opportunity to revamp how we do our business.

Unfortunately, at that point I was not completely sure what post-production meant. The thought of project managing the implementation of a media asset management (MAM) system for Lifetime suddenly seemed very daunting. Panic spread throughout me 
and I hoped no one noticed that I was completely lost.

Fortunately my boss did notice and after the meeting, he assured me that even though he had been on the project a few weeks that he was still jotting down terms for future Google searches and only understood half of what was being discussed. At that point, I could only dream of getting to that level.

\section{FAST-FORWARD}

Fast-forward a year to August 2005 and I'm back in a meeting. This time, I'm the one talking and I'm serving up acronyms like an IT version of Roger Federer. The meeting is about plans for our first end-to-end test of the system.

How did we get from the concept of MAM to being on the verge of testing a working system that can ingest a tape, convert it to a $50 \mathrm{Mb}$ IMX file that we can quality control (QC), archive, move into an Avid editing system, and transcode for on-air? Well, it is a story that involves buried treasure, pirates, a beautiful princess, and a dashing hero... Not quite. Actually, it involves the more mundane elements of project planning, vendor management, change management and taking a chance on new technology.

\section{STRUCTURING THE PROJECT}

Out first goal was to create a structured project. We knew upfront that no one had developed the exact system we wanted. It was going to be a complicated project with multiple vendors developing software and hardware. The only way we could manage this project would be to employ standard IT project management practices, but without overwhelming the broadcast team with an overly formal and bureaucratic process.

The first phase of the project was to incorporate the functions performed at our Astoria location into the MAM system:

- Ingest/QC: Ingest is the process of converting tape content into the desired high-resolution file format (for editing) and low-resolution proxy format (for producer review, annotation and approval). QC is the process of reviewing the tape for technical issues, violations of standards and practices (eg excessive nudity and violence) etc.

- Long form reformatting: Editing movies and episodes to conform to our segment sizes and standards and practices.

- Closed captioning: Extracting closed captioning information and merging closed captioning files with the content.

- Promo tagging: Taking promos and adding voice-overs and graphics that announce when a program will air.

- Dubbing: Creating tapes for distribution to the outside world, such as closed captioning agencies.

- Enabled desktop: The window into the MAM system that is used for administration, proxy viewing, annotation, review and approval. In the future, this is where clip sequences can be generated and EDL sent to the Avid for editing.

- Transfer to on-air: Converting the highresolution content to our on-air format and moving it to the automation system.

Promo development, which takes place in our midtown office, will remain in place and will be implemented in the second phase. The first phase of implementation, however, still has to account for promo development, so that the second phase will only require 
additional processes and not system changes.

In addition, the process for handling spots will remain untouched. As it is, about half of the commercials we air are sent to us as files via satellite or the internet. Bugs, those little graphics that pop-up on the bottom of the television screen, will be incorporated into the MAM system in the future. We also excluded the incorporation of graphics, voice-overs and music from the first phase. However, we already have people lobbying to put voice-overs into the MAM system. Keeping the scope from expanding is not always easy.

With the scope of the project defined, the next step was to identify the key players on the project. On the highest level, we formed a steering committee that meets on an ad hoc basis to discuss strategy and coordination between the different departments. The committee has representatives from broadcast operations, engineering, on-air, postproduction, promo development, applications development and IT infrastructure. The steering committee represents a real blend of IT and broadcast talent. The CIO who leads the committee is in charge of both IT and broadcast.

Having one person in charge of IT and broadcast has proven to be very helpful with the coordination of both groups. As broadcast technology requires more software and adopts IT standards such as TCP-IP, the lines between the two groups get blurrier. We still have a long way to go in terms of organizing and coordinating the two groups, but having one person in charge is a major step.

It was with the perspective of joint participation of IT and broadcast in the
MAM project, that we created a core team dedicated to the development of the MAM system. We wanted a group that was independent of broadcast and IT, but was able to understand and incorporate both perspectives. I am responsible for project management. In addition, we have a business analyst, an IT engineer and a broadcast engineer. We meet weekly with the head of broadcast and the CIO to present a formal status report. In addition, we have easy and unfiltered access to both at any time.

Despite the level of expertise in the group, we still need to work closely with broadcast operations, postproduction, IT and broadcast engineering. We have formed task forces to evaluate workflow, technical requirements and design. Their participation is crucial to the success of the project. In addition, it helps to keep them informed and involved in the project so that they feel ownership and buy-in. We have been fortunate to have a user community that is technically savvy and proactive about participating in the project.

With the teams in place, we were able to create a high-level project plan. The next challenge was to select our vendors. We felt that the most critical component was the MAM software as it would be interfacing with every aspect of the system.

\section{MAM VENDOR SELECTION}

To make our vendor selection, we had to employ our project management techniques to formally develop our requirements for the system components, functionality and integration points. The MAM system was expected to do a lot more than just 
store the content and the related metadata. We met with the task forces and reviewed and documented their current workflows, their data requirements, communications, and technical and equipment needs. From this we developed a list of systems that were required and functionality that had to be supported and developed about 200 requirements for the DAM system.

We created a list of MAM providers based on word of mouth, web research, our own knowledge and other vendors' recommendations. We then developed an RFP, where we described the system we needed. As we culled the number down, we set up Q\&A and demo sessions to review the vendors' offerings. Finally, we selected Venaca and their system called S3.

We realized that there were factors in addition to our specific system requirements that were critical to our vendor selection.

We wanted the system to support High Definition (HD) even though we would only support Standard Definition (SD) in the first phase. We did not want to introduce the high bandwidth requirements and uncertainty associated with a new technology like HD, at the same time as introducing MAM technology.

The vendor needed to support IMX and MXF standards. Although, we later found out that MXF was really a collection of standards, rather than a fixed standard.

No system provided a complete endto-end solution. Therefore, we wanted a vendor with a strong development team that was very knowledgeable about IT and broadcast technology. They had to guide us with the selection of vendors for encoding and decoding content, archive storage and SAN storage. Further, the vendor would have to create an integrated system that linked these components along with a nonlinear editing (NLE) system and the broadcast system. We preferred the vendor to have a local presence, because we needed to work closely with them on the design and testing of the system.

The MAM interface is a lower priority than the technology piece. Any upgrades on the interface will be relatively simplistic and lower priority compared with the technical hurdles of encoding and viewing the file in realtime, transferring the file to Avid, and transferring it to on-air. We needed a simple and clean interface. However, it is important that the system supports flexible and robust searching. The ability to search and locate content is critical to reusing content effectively.

Some of the core system requirements are:

- to generate a $50 \mathrm{Mb}$ IMX file from DigiBeta

- to generate low-resolution proxies that can be viewed on the desktop;

- that content can be viewed and annotations noted against it;

- to extract closed captioning;

- to provide robust search functionality;

- to move content to a non-linear editing system;

- to move content to on-air;

- to archive the content;

- to create dubs on tape from the IMX file;

- to enable administrators to:

- customize screens;

- add metadata fields;

- create a data dictionary and thesaurus. 
We also did our due diligence researching Venaca. We spoke with other customers, looked at the financial state of the company, and researched its growth in the past and its potential for growth in the future.

By January 2005, we created a base contract outlining the deliverables and requirements for status reporting and notification of issues. We also ensured that the code would be held in escrow. We specified that we would attach statements of work (SOW) for the contract, outlining our specific requirements. The SOW not only specified the functionality required, but also the performance criteria and quality criteria. Initially we agreed on a daily rate per developer, but as we further refined the contract we agreed on a fixed price contract for software license fees and development.

\section{DESIGN}

The core principle for the overall design was to build a multi-vendor, modular system, where we would have the ability to swap vendors in and out. The modules aligned with the functional breakdown of the system:

- QC/ingest station;

- central storage;

- archive;

- dubbing;

- editing system;

- enabled desktop; and

- transfer to on-air.

Having selected the MAM vendor, the next big decision was to select the vendor who would provide the ingest/encoding system. We had some very definite requirements. We wanted to generate a $50 \mathrm{Mb}$ IMX file for editing and an
MPEG-1 (between 1.5 and 2.0 Mb) proxy file. We wanted to be able to view the video from the file as it was being written to disk (decode while encode).

Again, no one vendor had everything we wanted. Ultimately, we went with Optibase, which had the ability to generate IMX 50 files. Optibase developed an ingest station for us. The ingest station has Optibase cards for creating the prod resolution file and the proxies. It uses a Vela card for the decode function.

Optibase had to modify their cards to handle four channels of embedded audio. Only a few desired features were put off for a future phase. For example, this version of the ingest station will not allow audio scrub of the high-resolution file. The SD Vela card does not provide this feature, so Optibase will later incorporate Vela's HD/SD card that does provide this feature. We had decided, primarily because of cost, that all of our encoders and decoders support $\mathrm{SD}$ and not HD. We will upgrade to $\mathrm{HD}$ in the future.

S3 manages the ingest/QC process. It automatically generates key frames that are based on specified time intervals or scene change detection. During ingest, the QC operator will view the video in real-time and mark annotations against a time code, using S3. These annotations will indicate technical problems with the video or audio, or indicate violations of our standards and practices regarding nudity, violence and foul language. The operator will also be able to jog and shuttle through the completely ingested video and make further annotations against time code.

In addition, S3 captures the closed captioning information during ingest and stores it as an annotation. We have 
commissioned Venaca to add the ability to generate a closed captioned file. This file will be in a format specified by another vendor, SoftNI, which has a system that we will use to read and generate various closed caption file formats used by closed captioning agencies.

We will also be able to generate a closed caption file on the on-air transcoders. In addition, the transcoder will take a closed caption file in the SoftNI format and merge it with the corresponding video file. However, the main function of the transcoders, which are Windows machines, is to take the IMX file and transcode it into a 12-15 Mb Long GOP file.

As on-air is considered sacrosanct, we want to isolate it from the rest of the network. The output of the transcoders is directed through a firewall to an Omneon catch server. The Omneon catch server is in a no man's land between two firewalls. One firewall is between the post-production environment and the catch server. The ingest stations, dubbing stations, SAN, archive, enabled desktop, the Avid gateway, Avid and transcoders to on-air are considered to be post-production.

The other firewall is between the catch server and the on-air network. This firewall isolates the on-air environment from the rest of Lifetime's network. Files can only be pulled from the on-air side and not pushed into the on-air side. The transcoders will output a video file, two audio channels, a QuickTime reference file, and an XML file with the content's metadata. These files are all pulled into the Harris automation system. The metadata specify the title, the code and other identifying characteristics of the content.
In addition, the metadata lists the segments' start and end time-codes. One issue we still need to address is defining a content ID that can be used in all Lifetime systems.

We also need an expandable, highspeed, high-storage robotic archive that could hold up to five years of production resolution SD content, and backup the proxies and the on-air resolution content. We selected ADIC's Scalar $10 \mathrm{~K}$ archive with $500 \mathrm{~Tb}$ of storage.

Choosing an editing system was one of the bigger challenges. Ultimately we selected Avid because of its robust functionality, its roadmap to support MXF and to increase its storage capacity, and because it is a de facto standard with a large installed base and a large pool of editing talent that knows how to use the system.

Avid have also opened up their technology, providing an API that will allow us to retrieve our encoded files, wrap them with Avid-compatible OP Atom wrappers, load the files into bins and pass metadata to the Avid. In turn we will be able to export a file out of Avid, flatten it, unwrap the file, register it into the MAM along with the metadata from the Avid, generate a proxy and archive the content. These functions are run on an Avid gateway, which is a Windows machine.

Initially we thought of using the Avid Unity as our central storage, but it did not scale to the size we required. We estimated we would need $9 \mathrm{~Tb}$. We will keep the proxies on the central storage and the files moving through the storage. To improve the performance of retrieving the proxies, we decided to keep them on disk rather than archive them. 
In addition, we wanted to maintain our modular design concept by keeping the central storage independent of the editing system. The central storage acts as the switching station for the content between all the other components. It also keeps the other components isolated from and independent of each other.

We selected a $9 \mathrm{~Tb}$ EMC SAN with StorNext hierarchical storage management. The SAN has to support several concurrent flows of data including the proxies that are read from the SAN by the enabled desktops.

In addition, there is the throughput of 12 ingest stations. The stations will write the content to the SAN and read it back. The content will be staged on the SAN until it is routed from the SAN to the robotic archive and possibly to the two Avid gateways or to the three on-air transcoders.

Two Avid gateways will be pulling content from the robotic archive via the SAN and pushing content from the Avid to the SAN and out to the archive or on-air transcoders. Three onair transcoders will be pulling content from the archive via the SAN. Nine dubbing stations will also pull content from the archive via the SAN. The dubbing stations are Windows machines with a Vela card for decoding the IMX file to SDI, which is routed to a tape deck. All components reading and writing to the SAN are attached via HBA fiber cards.

The enabled desktops are on the IT network, which run through our CISCO switches. The S3 application server (Windows server) is fiberattached to the SAN and connected to the ethernet network. The proxies are pulled from the SAN through the application server to the enabled desktops. We have not decided whether we will stream or download content. The enabled desktops are workstation windows PCs that are used by producers to view proxies, make clip reels and make annotations against the content.

Figure 1 provides an overview of the system design.

Once the design was complete, we created a more detailed project plan specifying the timeline for the development of the system. As we developed the system, we discovered items that had to be incorporated into the design. For example, we needed to supplement the Avid batch-back function to locate files properly, using S3's database, and load them back into the Avid. We also added the ability to flatten edited files on the Avid gateway, thus offloading the Avid workstation. We also added a faster logger screen to annotate the content during ingest. We expect to add more features as we test the system and work with prototype workflows.

\section{CONCLUSION}

Design of the system and its components was completed in May 2005. Final specification and selection of equipment was completed in July. With the users' participation we have already documented how the current system works and we have created a proposal for a future high-level workflow. We also developed a metadata model based on the workflow analysis, as well as other companies' metadata models and standards like Dublin Core.

We incorporated feedback from Venaca and the data requirements for on-air and for Avid. We also added fields to reflect the current state of the 


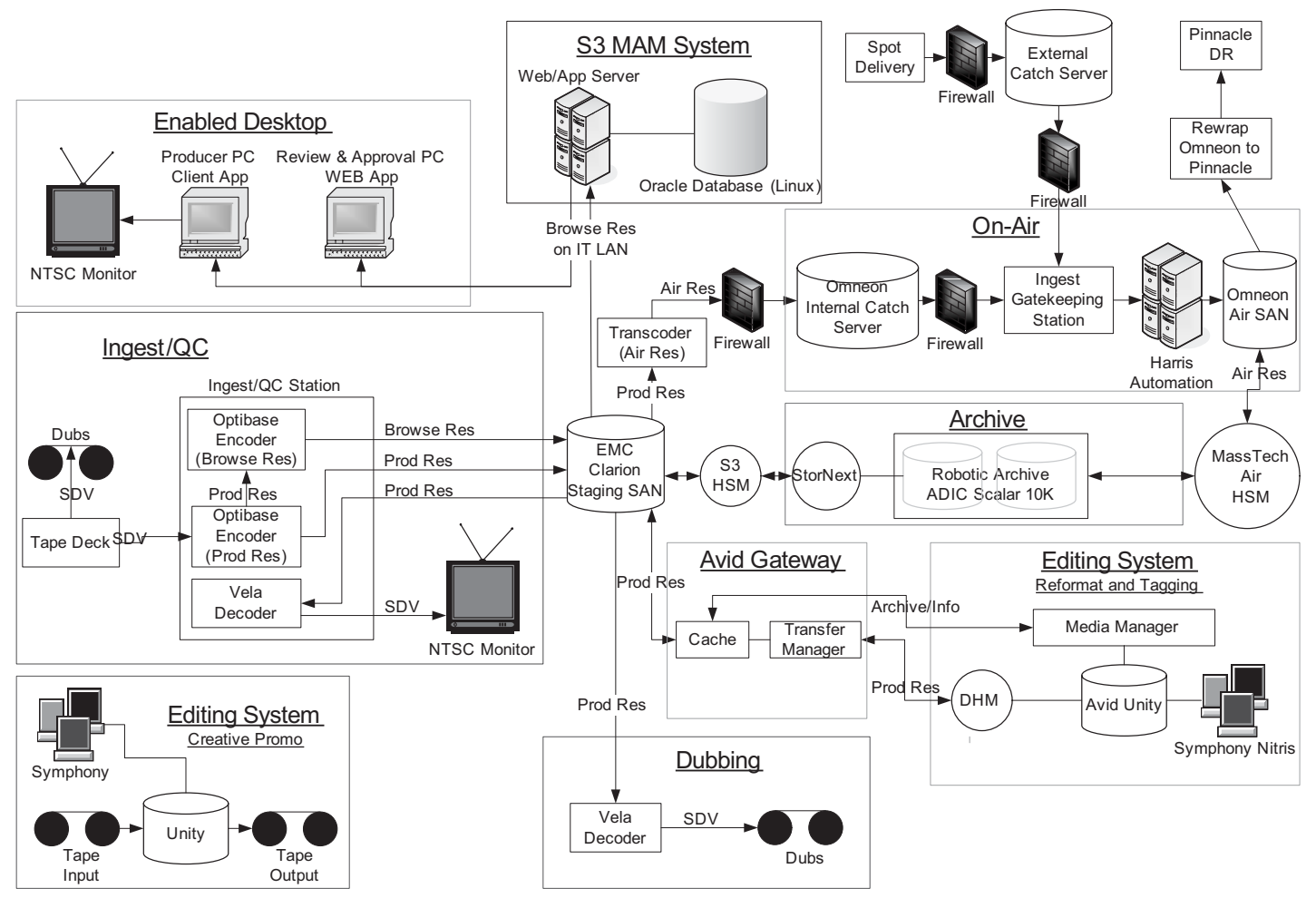

Figure 1: MAM architecture data flow

content and where it is in the workflow. However, we want to be frugal with the number of fields in the model in order to keep the data input and maintenance reasonable.

As of August 2005 we were in the process of building two test beds. The first was to evaluate the system for robustness and performance and to validate the movement of the files from ingest to on-air and all the steps along the way. This is a technical test of the hardware components and the software that connects the components together.

We met with our task forces and worked with them to create test plans, using standard test plan templates. We also specified equipment, such as scopes and meters, so we can objectively measure the quality of our files and monitor them as they move through the system.
Unfortunately there were delays in getting hardware. In turn, this affected the development and vendor testing that was dependent on having the hardware available. Some equipment didn't come in, so we had to borrow equipment. We also had problems with air conditioning and clean power at our test site. There was last minute trouble-shooting as this was the first time we have built the system end-to-end.

In the next two weeks construction of the test bed was due to be completed. In total, it took about six weeks to get it completely up and running. The major development was completed by the end of August. We estimated four to six weeks of testing starting in September. We were about two months behind our original plan, but we allotted sufficient time for slippage.

The second test bed started operation 
in September and continued for the rest of the year. We compared the MPEG-1 proxies at different bitrates, ranging from 1-2 Mbps, to ensure that we had at least VHS quality and minimal bandwidth requirements.

We also tested content generated from different sources, such as video and film, with different aspect ratios and quality. We have already fixed a problem with closed captioning on content originating from film converted using 3:2 pulldown.

In addition, this test bed will be used for evaluating screens and workflow. It is in this test bed that we will get a sense of how the implementation of the

MAM system will impact the way people will work in post-production. So far, we have concentrated on solving technical issues and we have not addressed the changes to people's jobs. To gain the efficiencies expected, we can't just provide new tools, but have to rethink the whole process. For example, we want to reduce the number of Excel spreadsheets used and rely instead on reports and searches off the MAM system.

People's jobs will change and we will re-evaluate who performs what roles. For example, we need to define the role of librarian (or archivist), who will ensure the quality of the data. We need someone to control the movement of content to Avid and to on-air.

The changes to people's jobs combined with moving the facility creates a lot of angst for the staff, who are concerned about their futures. Communication is critical. It has been important to involve the staff in system design, screen design, workflow design and testing. Not only does this give the staff a sense of ownership, but they are a technically savvy group with a strong desire to be involved in the system design and who offer a lot of insight and good ideas. We will continue to seek their input on workflow.

In 2006 we will be going live with the MAM system at our new location. In that time we must resolve several issues, in addition to finalizing the detail workflows.

- We need to define a universal ID for the content in the MAM and the on-air automation system.

- We need to determine the retention policy on the robotic archive.

- We have to finish our plan for disaster recovery.

- We have to develop a process for locating content on the MAM and the tape library.

- We have to train the staff.

- We have to plan the implementation of the MAM system at the new facility. This preparation includes ingesting content into the new system ahead of time and proving out the installation at the new facility.

- Venaca has to complete closed caption processing, flattening of the edited file, and audio scrub on the proxy.

We expect that we will be working out the kinks in the system for three to six months after production implementation. My group will be responsible for support and will coordinate with the IT and broadcast engineering groups. In particular, I am concerned about the ability of the system to play, jog and shuttle through the large files smoothly and whether the system will be robust and bulletproof, or if it will need constant babysitting. 
Once the first phase is complete and stable there won't be much time for reflection, as we will be working on the next phases. First, we will bring promo development into the MAM system. Then we will incorporate the library system into the MAM and integrate the MAM with ScheduALL, the system we use to book our QC, editing and dubbing sessions. In addition, we want to interface to our rights, program scheduling and advertising sales systems. Then we want to implement a workflow engine. Once this is done, we can all live happily ever after. 